Supporting Information:

\title{
Band sharpening and band alignment enable high quality factor to enhance thermoelectric performance in $\boldsymbol{n}$-type $\mathrm{PbS}$
}

Yu Xiao, ${ }^{1}$ Dongyang Wang, ${ }^{1}$ Yang Zhang,,${ }^{2}$ Congrun Chen, ${ }^{3}$ Shuxuan Zhang, ${ }^{3}$ Kedong Wang, ${ }^{3}$ Guangtao Wang, ${ }^{4}$ Stephen J. Pennycook, ${ }^{2}$ G. Jeffrey Snyder, ${ }^{5}$ Haijun Wu, ${ }^{2, *}$ and Li-Dong Zhao ${ }^{1, *}$

${ }^{1}$ School of Materials Science and Engineering, Beihang University, Beijing 100191, China

${ }^{2}$ Department of Materials Science and Engineering, National University of Singapore, 9 Engineering Drive 1, Singapore 117575, Singapore

${ }^{3}$ Department of Physics, Southern University of Science and Technology, Shenzhen 518055, China

${ }^{4}$ School of Physics, Henan Normal University, Xinxiang 453007, China

${ }^{5}$ Department of Materials Science and Engineering, Northwestern University, Evanston, IL 60208, USA 


\section{Experimental details}

Material synthesis: Raw materials, $\mathrm{Pb}$ particle (99.999\%, Aladdin element, China), S chunk (99.99\%, Aladdin element, China), Sb bulk (99.999\%, Aladdin element, China), Sn bulk (99.99\%, Aladdin element, China), and Te bulk (99.99\%, Aladdin element, China) were loaded into silica tubes with nominal compositions, flame-sealed at a residual pressure below $\sim 10^{-4}$ Torr, slowly heated to $1323 \mathrm{~K}$ in $24 \mathrm{~h}$ and maintained at this temperature for $6 \mathrm{~h}$. The furnace was shut down and cooled to room temperature. The obtained ingots were ground into powders and sintered by spark plasma sintering (SPS-211Lx) at $923 \mathrm{~K}$ for 15 min under an axial compressive stress of $50 \mathrm{MPa}$, resulting in highly densified disk-shaped samples.

Structural characterization: The powder X-ray diffraction (PXRD) patterns were obtained with $\mathrm{Cu} K \alpha(\lambda=1.5418 \AA)$ radiation in a reflection geometry on a diffractometer operating at $40 \mathrm{KV}$ and $20 \mathrm{~mA}$ and equipped with a position-sensitive detector. The calculation of lattice parameter was performed on the PXRD data with software package Materials Analysis using Diffraction (MAUD). (Scanning) transmission electron microscopy (TEM/STEM) and energy dispersive x-ray spectroscopy (EDS) studies were conducted using a JEOL ARM200F atomic resolution analytical electron microscope installed in the National University of Singapore equipped with a cold field-emission gun, a new ASCOR 5th order aberration corrector and an Oxford X-Max 100TLE X-ray detector.

Hall measurements: Hall coefficients $\left(R_{\mathrm{H}}\right)$ were measured under a reversible magnetic field $(0.8 \mathrm{~T})$ by the Van der Pauw method using a Hall measurement system (Lake Shore 8400 Series, Model 8404, USA) at room temperature. Carrier density $\left(n_{\mathrm{H}}\right)$ was obtained by $n_{\mathrm{H}}=1 /\left(e \cdot R_{\mathrm{H}}\right)$, and carrier mobility $\left(\mu_{\mathrm{H}}\right)$ was calculated using the relationship $\mu_{\mathrm{H}}=\sigma \cdot R_{\mathrm{H}}$ with $\sigma$ being the electrical conductivity.

Band gap measurements: Refined powder was used to measure the optical band gap by Infrared Diffuse Reflection method with a Fourier Transform Infrared Spectrometer (IRAffinity-1S). The spectra was collected in the mid-IR (5000 450 $\mathrm{cm}^{-1}$ ) and the reflectance to absorption data was converted to estimate the band gap 
using Kubelka-Munk equation: $\alpha / S=\left(1-R^{2}\right) /(2 R)$, where $R, S$ and $\alpha$ are the reflectance, scattering amd absorption coefficients, repectively.

Scanning Tunneling Microscope (STM): The STM experiments were performed using an Omicron scanning tunneling microscope (STM) that operates at room temperature in ultrahigh vacuum. The detail of the experiment and STM measurement is as follows. Firstly, the bulk sample was prepared and heated to $523 \mathrm{~K}$ which kept for about $120 \mathrm{~min}$ to get a clean surface. Then, the bulk sample was transferred into the STM module after natural cooling to room temperature in ultrahigh vacuum (about $2 \times 10^{-10} \mathrm{mbar}$ ). The $\mathrm{W}$ tip is got by electrochemical corrosion, which is fixed in $\mathrm{Au}(111)$ and used for imaging and I-V spectroscopic measurements. Then when two regions were shown in the image, a line across the two regions was chosen. There were 30 points each line, and each point was taken 50 times to collect the I-V spectra. The I-V spectra were taken at $1.5 \mathrm{~V}, 50 \mathrm{pA}$, and $\mathrm{dI} / \mathrm{dV}$ spectra were obtained by directly differential the I-V spectra.

Thermoelectric transport properties: The obtained spark plasma sintering (SPS) processed pellets were cut into bars with dimensions $12 \times 3 \times 3 \mathrm{~mm}^{3}$ for simultaneous measurement of the Seebeck coefficient and electrical conductivity using Cryoall CTA instrument under a low-pressure helium atmosphere from 300 to $923 \mathrm{~K}$. The uncertainty of the Seebeck coefficient and electrical conductivity measurement was $5 \%$. The SPS pellets were cut and polished into a disk-like shape with sizes of $\sim 6 \mathrm{~mm}$ diameter and $\sim 2 \mathrm{~mm}$ thickness for thermal diffusivity $(D)$ measurements. The samples were coated with a thin layer of graphite to minimize errors from the emissivity method in Netzsch LFA457. The sample density $(\rho)$ was determined using the dimensions and mass of the sample and reconfirmed by Archimedes method. The specific heat capacity $\left(C_{p}\right)$ was estimated with the Debye model. ${ }^{1}$ The thermal diffusivity data was analyzed using a Cowan model with pulse correction. The uncertainty of the thermal conductivity was estimated to be within $8 \%$, considering all the uncertainties from $D, \rho$ and $C_{p}$. The combined uncertainty for all measurements involved in the calculation of $Z T$ was less than $20 \%$.

Density functional theory (DFT) calculations: First-principles calculations within 
density functional theory were performed using the projector-augmented wave (PAW) method $^{2}$, as implemented in the Vienna Ab-initio Simulation Package (VASP) ${ }^{3}$. The exchange-correlation interactions were calculated by the generalized gradient approximation (GGA) of Perdew-Burke-Ernzerhof (PBE) ${ }^{4}$. Plane-Waves cutoff energy of $450 \mathrm{eV}$ and the Monkhorst-Pack $5 \times 5 \times 5 k$-point mesh was adopted for all the calculations. The atomic positions were fully relaxed until the maximum residual ionic force is below $0.01 \mathrm{eV} \AA^{-1}$, and the total energy difference is converged to within $10^{-7} \mathrm{eV}$. To avoid the defect-defect interaction, a $2 \times 2 \times 2\left(\mathrm{~Pb}_{32} \mathrm{Te}_{32}\right)$ supercell with two $\mathrm{Sn}$ atoms in fourth nearest neighborhood and a $2 \times 2 \times 2\left(\mathrm{~Pb}_{8} \mathrm{Te}_{8}\right)$ supercell with one Sn atom were adopted to describe the $6.25 \%$ and $12.5 \%$ Sn doped PbTe, respectively. For supercell with defects, the band structure is different from the primitive cell and band unfolding is necessary. The unfolding has been performed using the BandUP code 5 . 

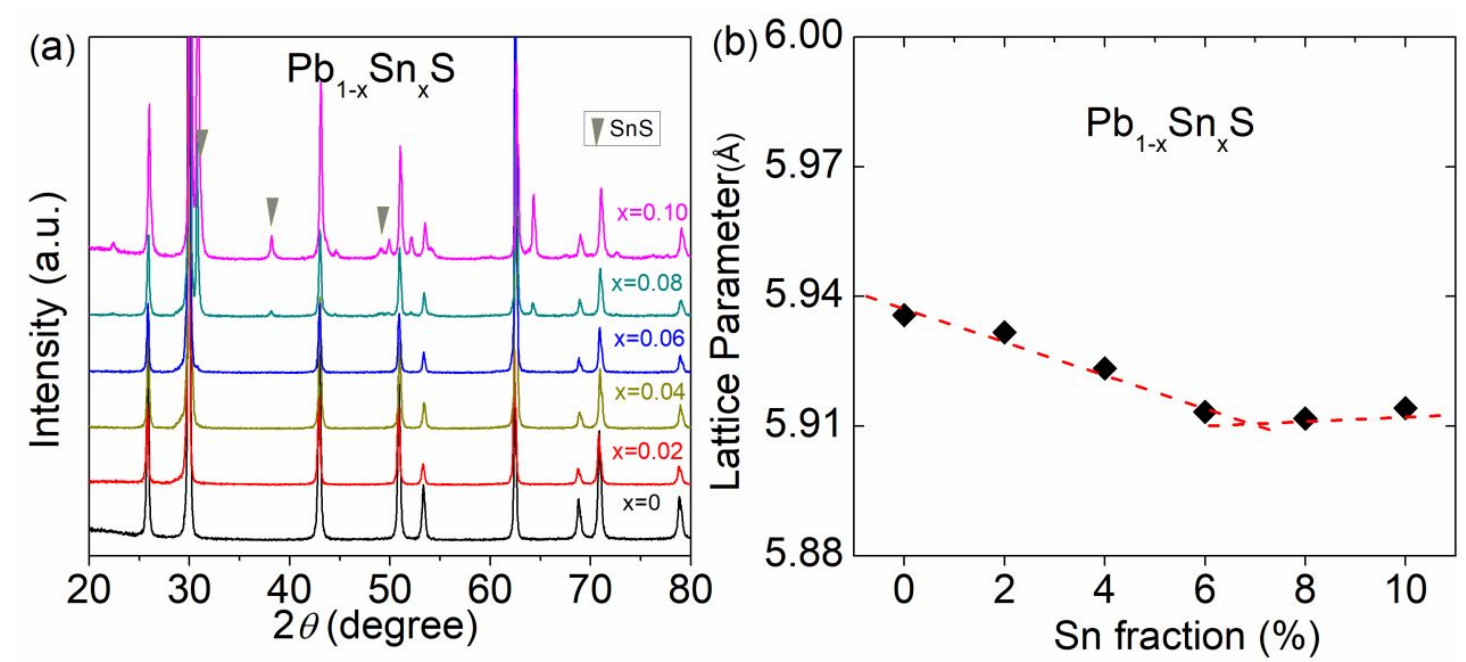

Figure S1. Phase identification of $\mathrm{Pb}_{1-\mathrm{x}} \mathrm{Sn}_{\mathrm{x}} \mathrm{S}(\mathrm{x}=0-0.10)$ : (a) PXRD patterns and (b) lattice parameter. 


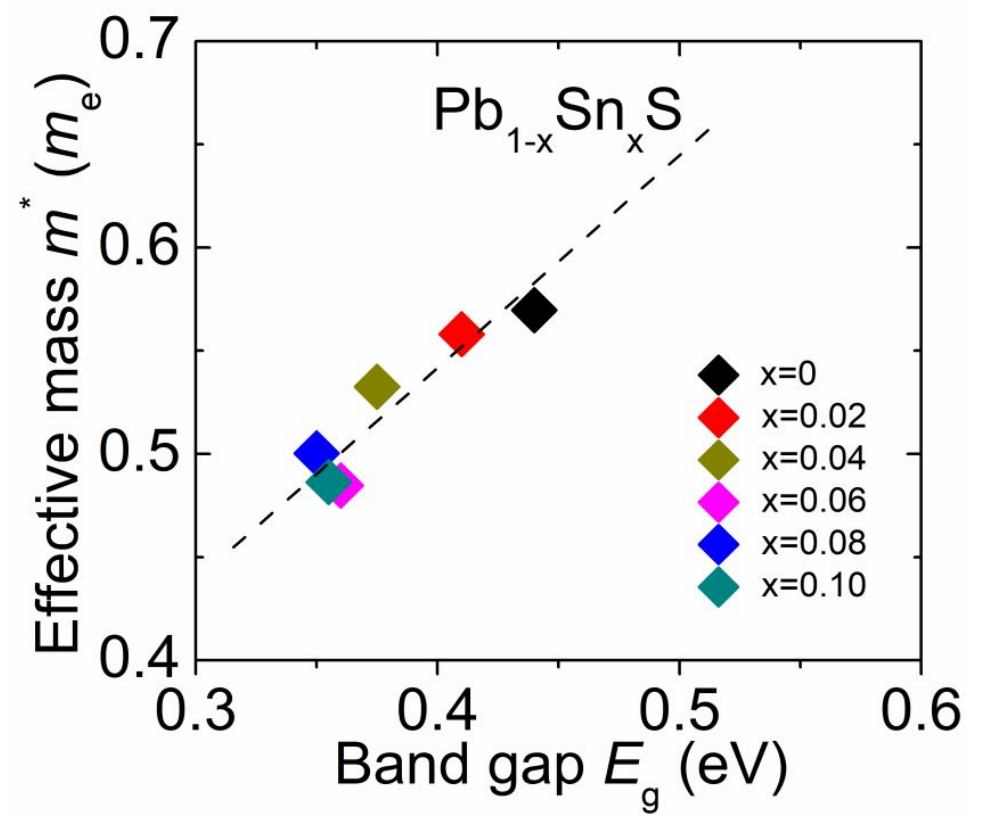

Figure S2. The relationship between band gap and effective mass in $\mathrm{Pb}_{1-\mathrm{x}} \mathrm{Sn}_{\mathrm{x}} \mathrm{S}(\mathrm{x}=\mathbf{0 - 0 . 1 0})$. 

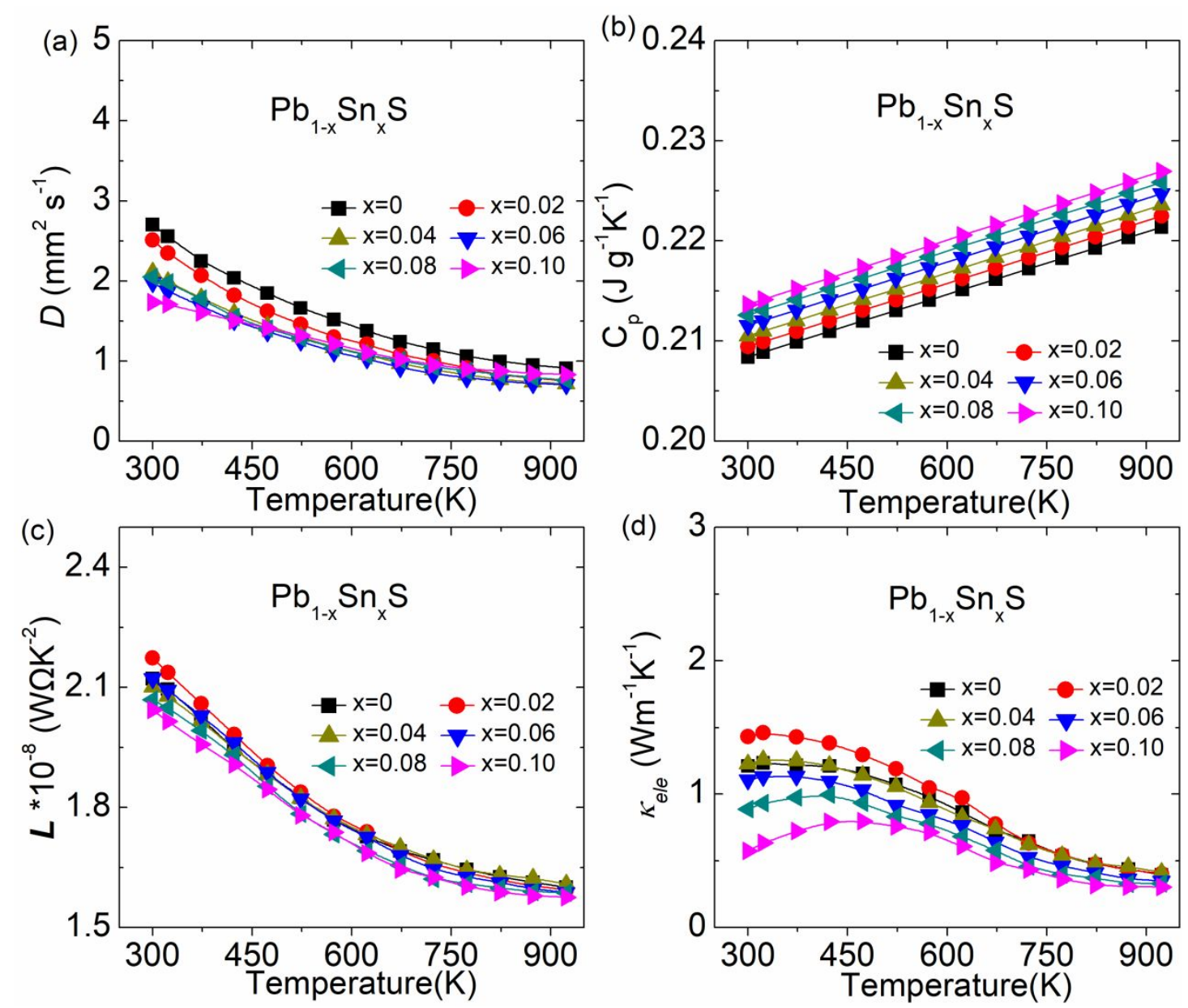

Figure S3. Thermal transport properties of $\mathrm{Pb}_{1-\mathrm{x}} \mathrm{Sn}_{\mathrm{x}} \mathrm{S}$ (x=0-0.10): (a) thermal diffusivity; (b) heat capacity; (c) Lorenz number; and (d) electronic thermal conductivity. 

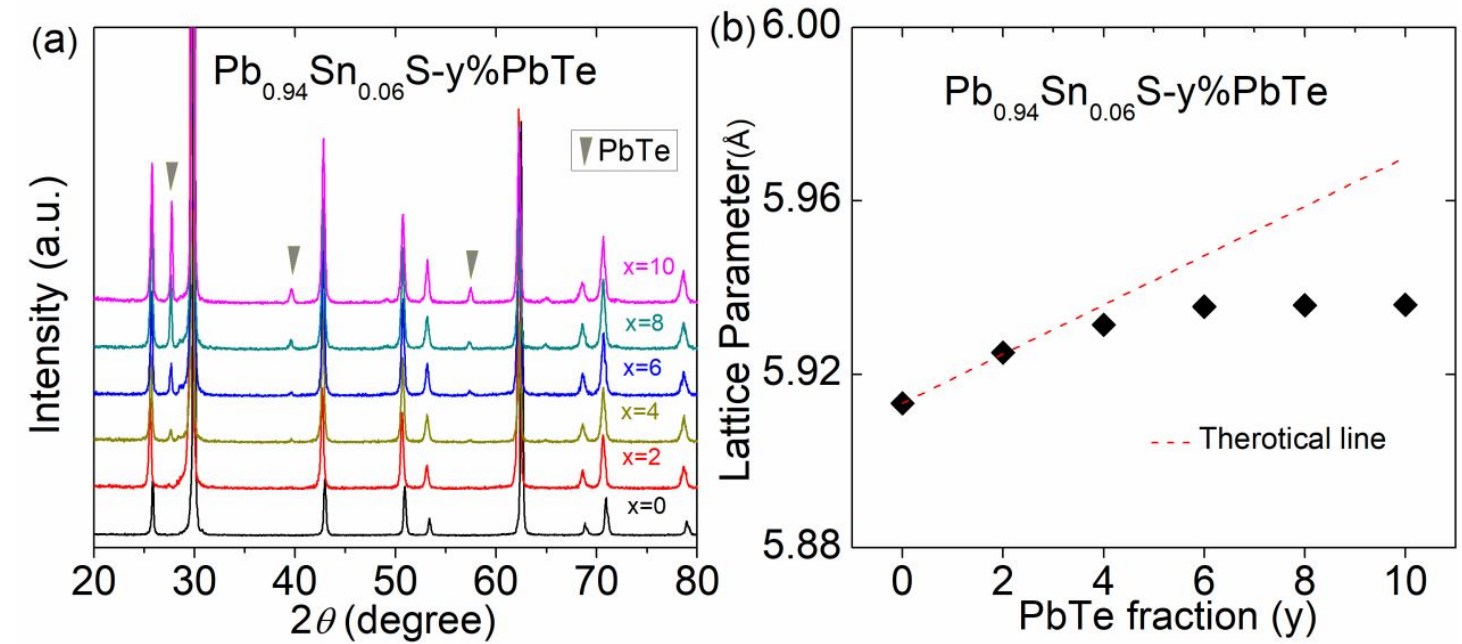

Figure S4. Phase identification of $\mathrm{Pb}_{0.94} \mathrm{Sn}_{0.06} \mathrm{~S}-\mathrm{y} \% \mathrm{PbTe}(\mathrm{y}=\mathbf{0 - 1 0})$ : (a) PXRD patterns and (b) lattice parameter. 

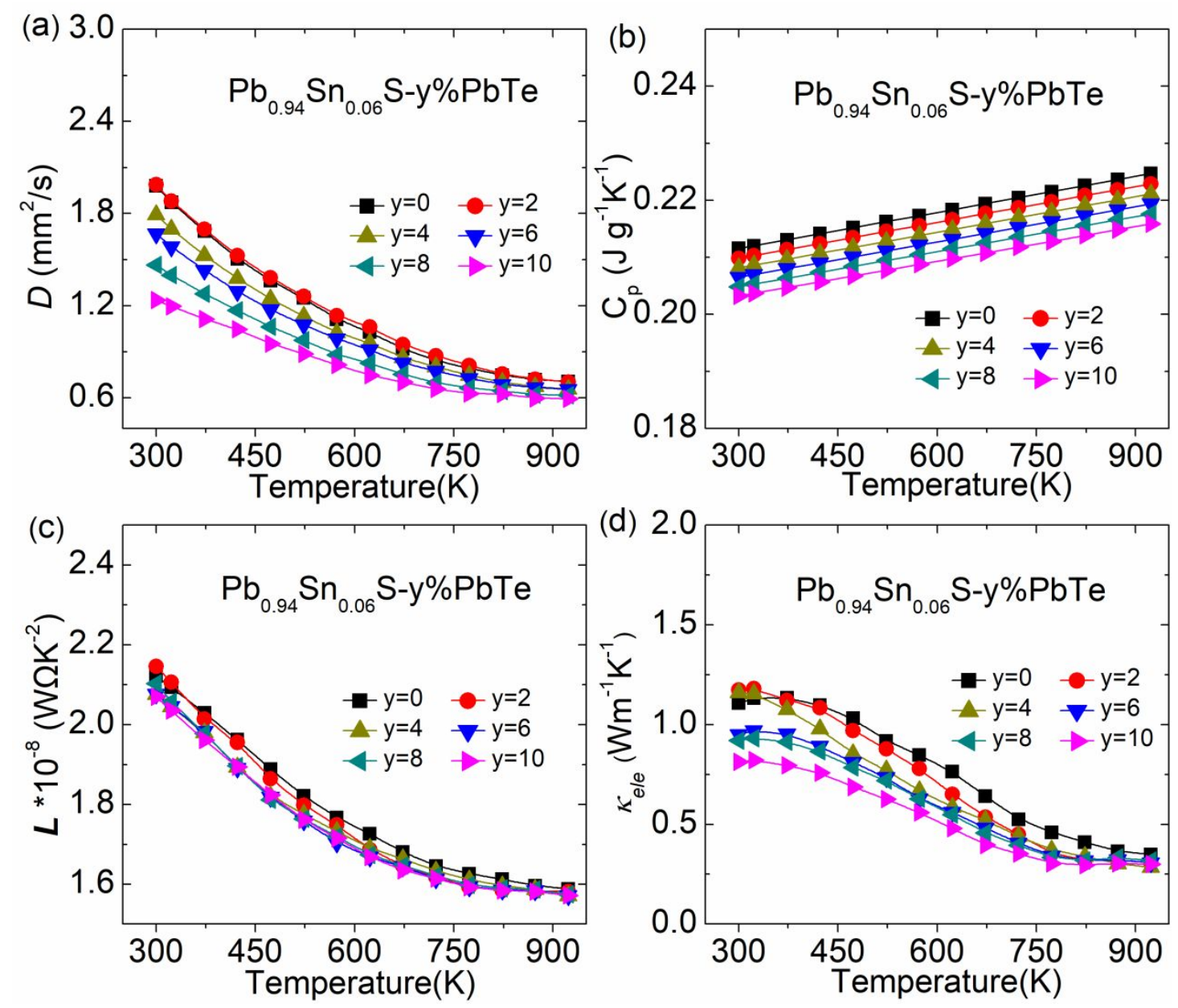

Figure S5. Thermal transport properties of $\mathrm{Pb}_{0.94} \mathrm{Sn}_{0.06} \mathrm{~S}-\mathrm{y} \% \mathrm{PbTe}(\mathrm{y}=0-10)$ : (a) thermal diffusivity; (b) heat capacity; (c) Lorenz number; and (d) electronic thermal conductivity. 

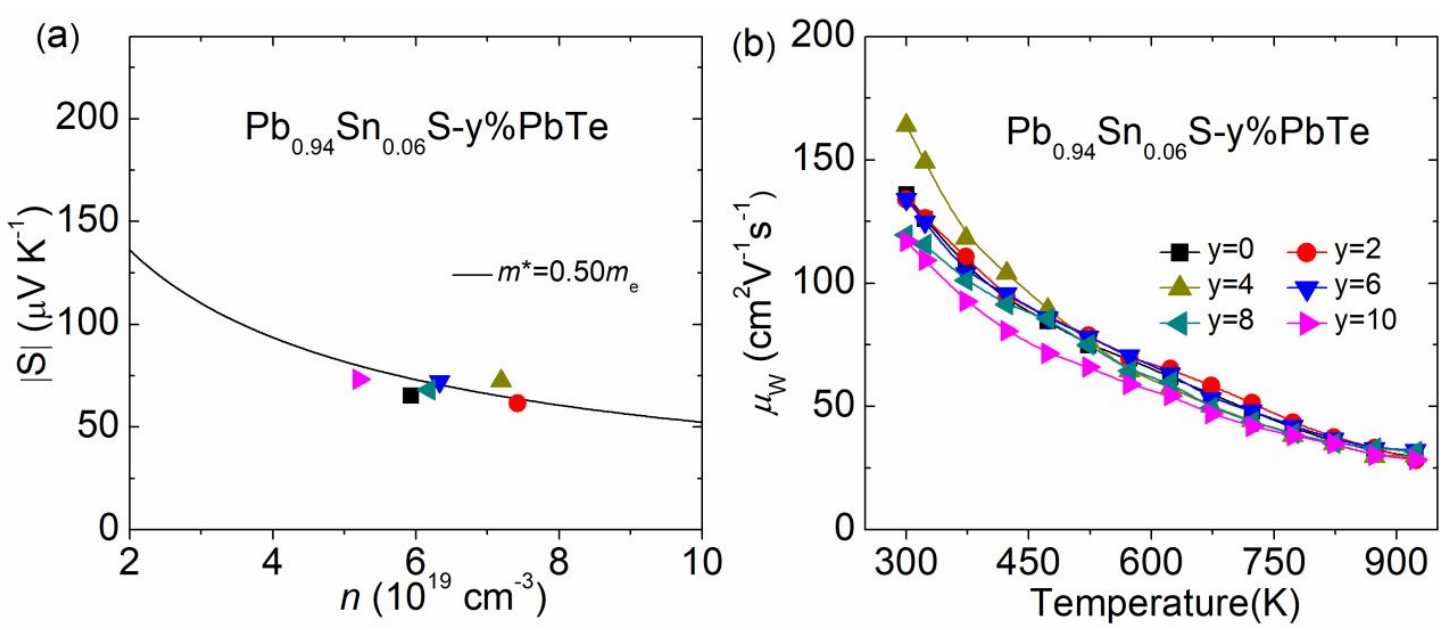

Figure S6. Electrical transport properties of $\mathrm{Pb}_{0.94} \mathrm{Sn}_{0.06} \mathrm{~S}-\mathrm{y} \% \mathrm{PbTe}(\mathrm{y}=\mathbf{0 - 1 0})$ : (a) Pisarenko relationship with effective mass $m^{*}=0.50 m_{\mathrm{e}}$ and (b) weighted carrier mobility. 


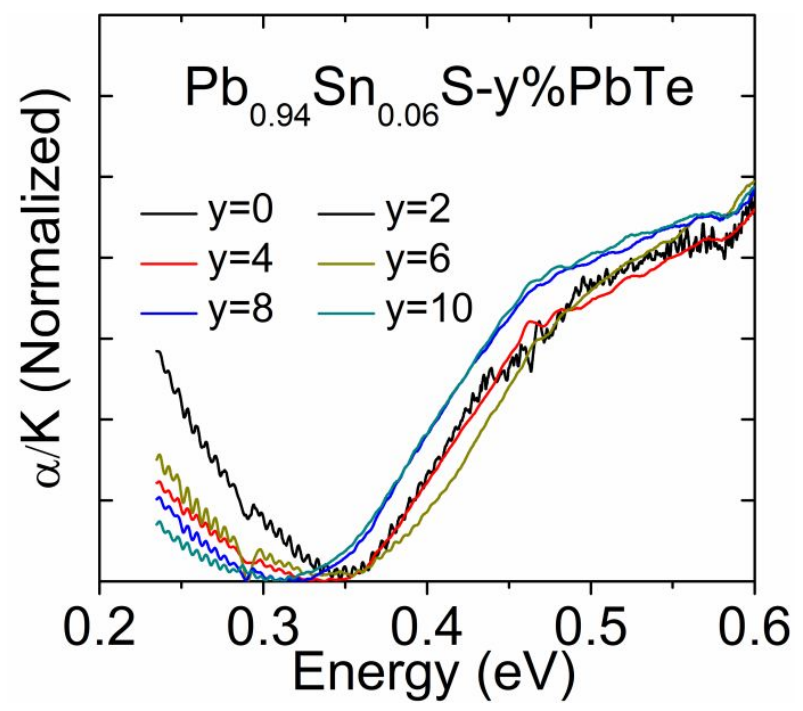

Figure S7. Optical band gap of $\mathrm{Pb}_{0.94} \mathrm{Sn}_{0.06} \mathrm{~S}-\mathrm{y} \% \mathrm{PbTe}(\mathrm{y}=\mathbf{0}-10)$. 
Table S1: Thermoelectric parameters of $\boldsymbol{n}$-type PbS-based materials in this work, including sample density $(\rho)$, carrier density $(n)$, Hall mobility $\left(\mu_{\mathrm{H}}\right)$, minimum lattice thermal conductivity $\left(\kappa_{\operatorname{lmin}}\right)$, maximum $Z T$ value $\left(Z T_{\max }\right)$ and average $Z T$ value $\left(Z T_{\text {ave }}\right)$ in 300-923 K.

\begin{tabular}{|c|c|c|c|c|c|c|}
\hline samples & $\begin{array}{c}\rho \\
\left(\mathrm{gcm}^{-3}\right)\end{array}$ & $\begin{array}{c}n \\
\left(10^{19} \mathrm{~cm}^{-3}\right. \\
)\end{array}$ & $\begin{array}{c}\mu_{\mathrm{H}} \\
\left(\mathrm{cm}^{2} \mathbf{V}^{-1} \mathbf{s}^{-1}\right)\end{array}$ & $\begin{array}{c}\kappa_{\operatorname{lmin}} \\
\left(\mathbf{W m}^{-1} \mathbf{K}^{-1}\right)\end{array}$ & $Z T_{\max }$ & $\begin{array}{c}Z T_{\text {ave }} \\
(300-923 K)\end{array}$ \\
\hline $\mathrm{PbS}$ & 7.25 & 7.56 & 157 & 1.07 & 0.84 & 0.48 \\
\hline $\mathrm{Pb}_{0.98} \mathrm{Sn}_{0.02} \mathrm{~S}$ & 7.28 & 8.85 & 155 & 0.82 & 1.06 & 0.58 \\
\hline $\mathrm{Pb}_{0.96} \mathrm{Sn}_{0.04} \mathrm{~S}$ & 7.15 & 6.34 & 192 & 0.72 & 1.05 & 0.60 \\
\hline $\mathrm{Pb}_{0.94} \mathrm{Sn}_{0.06} \mathrm{~S}$ & 7.05 & 5.93 & 183 & 0.77 & 1.07 & 0.60 \\
\hline $\mathrm{Pb}_{0.92} \mathrm{Sn}_{0.08} \mathrm{~S}$ & 7.07 & 5.17 & 172 & 0.88 & 0.94 & 0.57 \\
\hline $\mathrm{Pb}_{0.90} \mathrm{Sn}_{0.10} \mathrm{~S}$ & 7.02 & 4.54 & 128 & 1.02 & 0.88 & 0.52 \\
\hline $\mathrm{Pb}_{0.94} \mathrm{Sn}_{0.06} \mathrm{~S}-2 \% \mathrm{PbTe}$ & 7.08 & 7.42 & 153 & 0.80 & 1.02 & 0.61 \\
\hline $\mathrm{Pb}_{0.94} \mathrm{Sn}_{0.06} \mathrm{~S}-4 \% \mathrm{PbTe}$ & 7.05 & 7.19 & 162 & 0.74 & 1.11 & 0.63 \\
\hline $\mathrm{Pb}_{0.94} \mathrm{Sn}_{0.06} \mathrm{~S}-6 \% \mathrm{PbTe}$ & 7.10 & 6.33 & 150 & 0.71 & 1.22 & 0.69 \\
\hline $\mathrm{Pb}_{0.94} \mathrm{Sn}_{0.06} \mathrm{~S}-8 \% \mathrm{PbTe}$ & 7.10 & 6.18 & 147 & 0.62 & 1.30 & 0.72 \\
\hline $\mathrm{Pb}_{0.94} \mathrm{Sn}_{0.06} \mathrm{~S}-10 \% \mathrm{PbTe}$ & 7.15 & 5.20 & 157 & 0.61 & 1.22 & 0.71 \\
\hline
\end{tabular}

\section{REFERENCE}

1. Qin, B.; Wang, D.; He, W.; Zhang, Y.; Wu, H.; Pennycook, S. J.; Zhao, L.-D., Realizing high thermoelectric performance in p-type $\mathrm{SnSe}$ through crystal structure modification. J. Am. Chem. Soc. 2018, 141 (2), 1141-1149.

2. Blöchl, P. E., Projector augmented-wave method. Phys. Rev. B 1994, 50 (24), 17953-17979.

3. Kresse, G.; Furthmüller, J., Efficient iterative schemes for ab initio total-energy calculations using a plane-wave basis set. Phys. Rev. B 1996, 54 (16), 11169-11186.

4. Perdew, J. P.; Burke, K.; Ernzerhof, M., Generalized gradient approximation made simple. Phys. Rev. Lett. 1996, 77 (18), 3865-3868.

5. Medeiros, P. V. C.; Stafström, S.; Björk, J., Effects of extrinsic and intrinsic perturbations on the electronic structure of graphene: Retaining an effective primitive cell band structure by band unfolding. Phys. Rev. B 2014, 89 (4), 041407. 